\title{
The optical spacer - a simple device which extends the scope of indirect ophthalmoscopy
}

Figure 1 A prototype device showing fixation targets.

Tennent Institute of Ophthalmology, University of Glasgow D M I Montgomery

Correspondence to:

D M I Montgomery, FRCS,

31 Moray Place, Strathbungo,

Glasgow G41 2BL.

Accepted for publication 17 May 1991

$\mathrm{R}$

$\mathbf{P}$

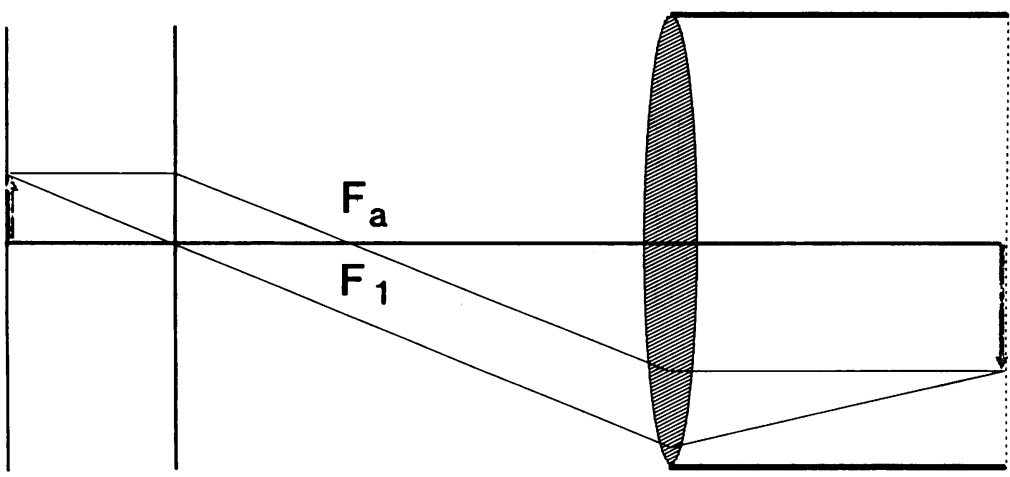

Optical Spacer

Figure 2 A transparent screen is positioned in the principal plane of the condensing lens by an optical spacer. The retina is imaged in this plane while targets introduced on to the screen are imaged on the retina. $P=$ principal plane. $F_{1}=$ first principal focus of lens. $F_{\mathrm{a}}=$ anterior focus of eye.

The optical spacer is a hollow cylinder which, when coupled to an indirect ophthalmoscopy condensing lens, enables a transparent screen to be positioned at the second principal focus of the lens (Fig 1). When the fundus is observed, the retinal image is formed in the plane of the screen and can thereby be accurately localised in threedimensional space (Fig 2). Each point on the retina in view forms an image on a corresponding point on the screen, while each point on the screen is in turn imaged upon, and therefore stimulates, its corresponding retinal point which it is seen to overlie. The ease with which stimuli may be introduced into the image plane is the key to several useful applications.

\section{Assessment of fixation}

If a patient is asked to look at a cross marked on the centre of the screen (Fig 3a) his fovea will be aligned with the cross unless his fixation is abnormal. The point and steadiness of fixation may therefore be rapidly assessed, for example, in a child with amblyopia or indeed in any condition where foveal function may be considered to be compromised.

\section{Fixation targets to facilitate indirect} ophthalmoscopy

A fixation target in the centre of the screen allows the patient's eye to be held steady while the posterior pole is examined. If the screen and lens are now rotated about their axis while the eye fixes on a target at the margin of the screen (Fig 3a), the smooth pursuit system is exploited to enable the fundus to be scanned in one continuous movement rather than as a series of disjointed, unpredictable saccades.

\section{Convenient recording of fundus lesions}

A lesion in the fundus may be accurately recorded simply by tracing the outline of its image directly on to an acetate sheet placed upon the screen. This record may then be detached and stored in the patient's case notes. At the next attendance the previously traced outline may be again superimposed upon the lesion. Any change in size will therefore become immediately apparent (Fig 3b).

\section{Assessment of distance visual acuity}

If standard Snellen test types are photographed at $6 \mathrm{~m}$ with a $35 \mathrm{~mm}$ lens and the developed film is then centred on the optical spacer screen located in the focal plane of a +28 dioptre condensing lens, the test types, when viewed through the lens, will subtend the same visual angle as their $6 \mathrm{~m}$ equivalents. A convenient, portable visual acuity test is thereby produced (Fig 3c).

\section{Visual field assessment with simultaneous funduscopy}

Since each point on the optical spacer screen corresponds to the retinal point imaged at that location, it is possible to map the central visual field under direct vision by 'pointing' at the retinal image with a target pin during ophthalmoscopy while the image is stabilised by a foveal fixation target. By this means functional defects may be readily correlated with the morphological appearance of the retina. The screen may have visual angle and meridional markings to facilitate comparisons with conventional visual field charts (Fig 3d). 
(a)

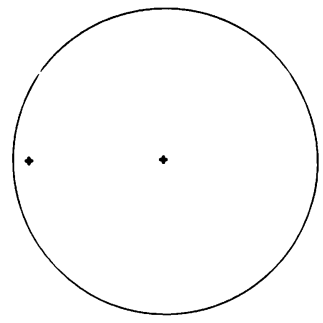

(c)

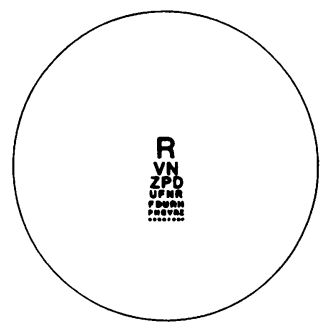

Figure 3 Selection of screens for use with the optical spacer. (a) Central target allows stabilisation of fundus image and assessment of fixation. Eccentric target enables fundus to be scanned in one continuous movement. (b) The outline of fundus lesions may be traced directly, allowing rapid recognition of change in size. (c) Portable distance Snellen acuity chart. $(d)$ The central visual field may be explored during ophthalmoscopy, allowing functional defects to be correlated with the morphological appearance of the retina.

Figure 4 The effect on image size of moving the condensing lens progressively further from the eye in ametropia.

$M=$ myopia $. E=$ emmetropia. $H=$

hypermetropia. $P=$ principal plane. $F_{1}=$ first principal focus of condensing lens. $F_{\mathrm{a}}=$ anterior focus of eye.

(Adapted with permission from Elkington AR, Frank HF: Clinical optics. Blackwell, 1984: 134.

(b)

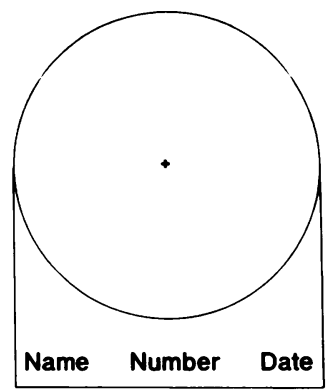

(d)

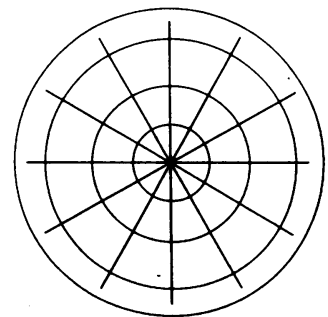

Accurate measurement of posterior segment structures and lesions

When the emmetropic eye is examined, because light rays emerging from the eye are parallel, the size of the image formed is independent of the distance of the condensing lens from the patient's eye. However, in ametropia the emergent rays are either convergent or divergent, and the position and magnification of the image will therefore be influenced by this distance (Fig 4). This effect becomes significant only at degrees of ametropia beyond about 3 dioptres (Fig 5), when it may be overcome by

MEH P
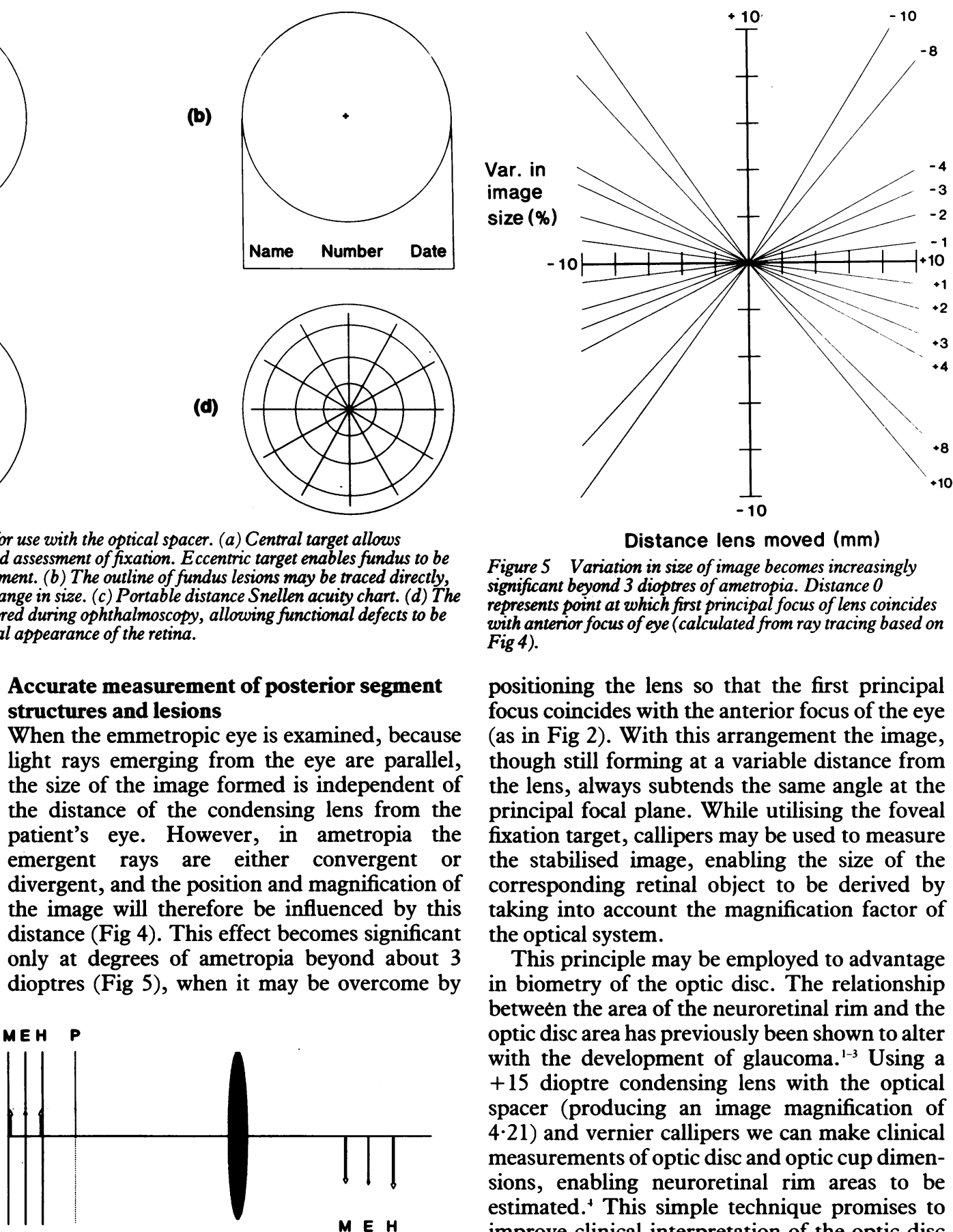

Distance lens moved (mm)

Figure 5 Variation in size of image becomes increasingly significant beyond 3 dioptres of ametropia. Distance $O$ represents point at which first principal focus of lens coincides with anterior focus of eye (calculated from ray tracing based on Fig 4).

positioning the lens so that the first principal focus coincides with the anterior focus of the eye (as in Fig 2). With this arrangement the image, though still forming at a variable distance from the lens, always subtends the same angle at the principal focal plane. While utilising the foveal fixation target, callipers may be used to measure the stabilised image, enabling the size of the corresponding retinal object to be derived by taking into account the magnification factor of the optical system.

This principle may be employed to advantage in biometry of the optic disc. The relationship between the area of the neuroretinal rim and the optic disc area has previously been shown to alter with the development of glaucoma..$^{1-3}$ Using a +15 dioptre condensing lens with the optical spacer (producing an image magnification of $4 \cdot 21)$ and vernier callipers we can make clinical measurements of optic disc and optic cup dimensions, enabling neuroretinal rim areas to be estimated. ${ }^{+}$This simple technique promises to improve clinical interpretation of the optic disc in glaucoma.

Pathological lesions at the posterior pole may be measured by the same method, while measurement in the more peripheral fundus requires in addition that the wide-field retinal magnification factor be taken into account. ${ }^{5}$

1 Airaksinen PJ, Drance SM, Douglas GR, Schulzer M. Neuroretinal rim area in early glaucoma. Am $\mathcal{F}$ Ophthalmol 1985 ; 99: $1-4$

2 Airaksinen PJ, Drance SM, Douglas GR, Schulzer M. Neuroretinal rim areas and visual field indices in glaucoma. $A m \mathcal{F}$ Ophthalmol 1985; 99: 107-10.

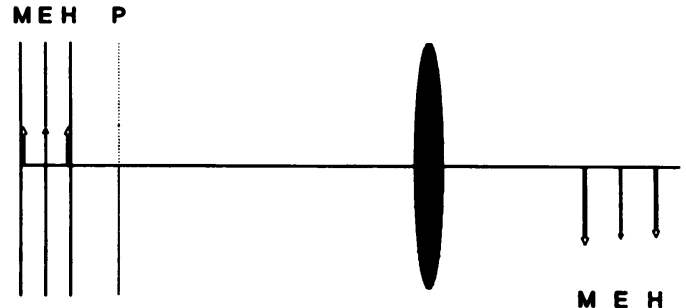

Airaksinen PJ, Drance SM. Neuroretinal rim area and retinal nerve fibre layer in glaucoma. Arch Ophthalmol 1985; 103: 203-4.

4 Montgomery DMI. Measurement of optic disc and neuroretinal rim areas in normal and glaucomatous eyes. A new clinical method. Ophthalmology 1991; 98: 50-9.

5 Holden AL, Fitzke FW. Image size in the fundus: structural evidence for wide-field retinal magnification factor. $\mathrm{Br} \mathcal{J}$ Ophthalmol 1988; 72: 228-30. 